\title{
Resistance gene cloning from a wild crop relative by sequence capture and association
} genetics

\begin{abstract}
Disease resistance $(\mathrm{R})$ genes from wild relatives could be used to engineer broad-spectrum resistance in domesticated crops. We combined association genetics with $\mathrm{R}$ gene enrichment sequencing (AgRenSeq) to exploit pan-genome variation in wild diploid wheat and rapidly clone four stem rust resistance genes. AgRenSeq enables R gene cloning in any crop that has a diverse germplasm panel.
\end{abstract}

Keyword: Agricultural genetics; Plant biotechnology; Plant molecular biology 